\title{
Predição computacional de alvos moleculares de um complexo metálico de rutênio com epiisopiloturina e óxido nítrico
}

\section{Computational prediction of molecular leishmania targets for a ruthenium metal complex with epiisopiloturine and nitric oxide}

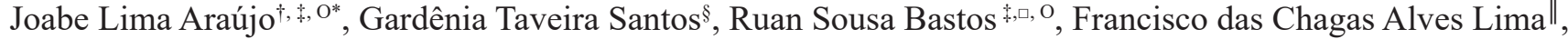 \\ Jefferson Almeida Rocha
}

Como citar esse artigo. Araujo,

J.L.; Santos, G.T.; Bastos, R.S.; Lima, F.C.A.; Rocha, J.A. Predição computacional de alvos moleculares de um complexo metálico de rutênio com epiisopiloturina e óxido nítrico. Revista de Saúde. 2020 Jan./Jun.; 11 (1): 42-48

\section{Resumo}

A Leishmaniose é uma doença infecciosa que ocasiona a morte de 26.000 a 65.000 pessoas anualmente, estima-se que no ano de 2019 houve 700.000 a 1 milhão de novos casos. Estes dados são preocupantes e está relacionado à falta de saneamento básico que favorece a proliferação dos vetores, além da ausência de medicamentos eficientes com mecanismos de ação alternativos e com menos efeitos colaterais. Em meio a essa necessidade de novos agentes inibitórios, este estudo teve como objetivo realizar uma predição computacional de alvos moleculares de Leishmania para um complexo metálico de rutênio com epiisopiloturina e óxido nítrico $\left(\right.$ Epiruno $\left._{2}\right)$. O processo de docking molecular foi realizado empregando-se o software Autodock Tools (ADT) versão 1.5.6. As proteínas alvos foram consideradas rígidas, enquanto que o Epiruno foi considerado flexível. A glicoproteína GP63 (1lml) representa mais de 1\% da proteína total do parasito tendo em vista que a $1 \mathrm{lml}$ é uma metaloprotease que predomina grupos funcionais em seu sítio ativo, torna-se um alvo atrativo em estudos de atividade inibitória. O docking molecular entre o Epiruno ${ }_{2}$ e a $11 \mathrm{ml}$ resultou na melhor conformação de encaixe deste estudo, com energia de $\mathrm{G}_{\text {bind }}{ }^{a}$ de $-8,05 \mathrm{Kcal}_{\text {.mol }}{ }^{-1}$ e uma constante de inibição de 1,26 $\mu \mathrm{M}$. Também foi observada a formação de quatro pontes de hidrogênio, demonstrando ser um forte candidato a fármaco antileishmania. Concluindo-se que o composto Epiruno 2 é clinicamente atrativo para estudos experimentais futuros ex vivo, in vitro e in vivo, pois seus resultados in sílico apresentaram boas interações moleculares para todas as proteínas alvo deste estudo.

Palavras-chave: Simulação de Acoplamento Molecular, Leishmaniose, Doença Negligenciada, atividade antileishmanial.

\begin{abstract}
Leishmaniasis is an infectious disease that causes the death of 26,000 to 65,000 people annually, it is estimated that in 2019 there were 700,000 to 1 million new cases. These data are worrying and are related to the lack of basic sanitation that favors the proliferation of vectors, in addition to the absence of efficient drugs with alternative mechanisms of action and with less side effects. Amid this need for new inhibitory agents, this study aimed to perform a computational prediction of Leishmania molecular targets for a ruthenium metal complex with epiisopiloturine and nitric oxide $\left(\right.$ Epiruno $\left._{2}\right)$. The molecular docking process was performed using the Autodock Tools (ADT) software version 1.5.6. The target proteins were considered rigid, while Epiruno, was considered flexible. The glycoprotein GP63 (11ml) represents more than 1\% of the total protein of the parasite, considering that $11 \mathrm{ml}$ is a metalloprotease that predominates functional groups in its active site, it becomes an attractive target in studies of inhibitory activity. The molecular docking between Epiruno and $1 \mathrm{lml}$ resulted in the best fit conformation in this study, with Gbinda energy of $-8.05 \mathrm{Kcal}$.mol-1 and an inhibition constant of $1.26 \mu \mathrm{M}$. The formation of four hydrogen bonds was also observed, demonstrating to be a strong candidate for antileishmania drug. In conclusion, the compound Epiruno ${ }_{2}$ is clinically attractive for future experimental studies ex vivo, in vitro and in vivo, as in silico results showed good molecular interactions for all the target proteins in this study.

Keywords: Molecular Docking Simulation, Leishmaniasis, Neglected Diseases, Antileishmanial activity.
\end{abstract}

\section{Introdução}

A Leishmaniose é uma doença infecciosa que ocasiona a morte de 26.000 a 65.000 pessoas anualmente, estima-se que no ano de 2019 houve 700.000 a 1 milhão de novos $\operatorname{casos}^{1}$. Estes dados são preocupantes, pois em pleno século XXI possuímos números elevados de casos de Leishmania, isso está relacionado à falta de saneamento básico que favorece a proliferação dos vetores, além da ausência de medicamentos eficientes devido à falta de interesse das indústrias farmacêuticas em produzir novas drogas com mecanismos de ação alternativos e com menos efeitos colaterais, pois grandes empresas farmacêuticas encerraram seus programas de pesquisa contra doenças negligenciadas e se concentraram em áreas terapêuticas potencialmente 
mais lucrativas ${ }^{2}$.

Isso ocorre devido ao público alvo, pois a doença se concentra em países subdesenvolvidos e não possuem poder econômico de investir e financiar tratamento para as pessoas diagnosticadas com a doença. Deste modo, cepas parasitárias se tornam cada vez mais resistentes e os medicamentos diminuem sua eficácia, além de serem potencialmente tóxicos ${ }^{3}$. Pois os fármacos Glucantime, antimoniato de meglumina e estiboglucnato de sódio (SSG), medicamentos de primeira linha contra a leishmaniose estão em uso desde a década de 1950 e não passaram por otimizações, em alguns casos a eficácia destes fármacos chega a apenas $10 \%$ como foi evidenciado em casos de Leishmania na índia ${ }^{4}$.

Isso mostra a necessidade de desenvolver novas substâncias com novos mecanismos de ação que apresente menos efeitos colaterais e alta eficácia. Associado a isso, temos estudos disponíveis na literatura sobre o uso de complexos metálicos para impulsionar a atividade biológica de compostos orgânicos em reação a uma proteína alvo, diversos medicamentos usados atualmente no tratamento de infecções por tripanossomatídeos possuem em sua composição metais, a exemplo do arsênico e antimônio ${ }^{5}$. No entanto, a compostos contendo outros metais, como prata, paládio, platina e rutênio que vêm apresentando resultados promissores em inibir proteínas-chave de Leishmania $^{6,7,8}$.

Um estudo realizado por Araújo et al. ${ }^{3}$ mostrou que um complexo de rutênio com epiisopiloturina e óxido nítrico (Epiruno $)_{2}$ obteve resultados atrativos de afinidade molecular para a proteína Nucleosidediphosphate kinase de L. major. Baseando-se nestes estudos, partimos da hipótese que o Epiruno ${ }_{2}$ apresente importante afinidade molecular para proteínas-chave de Leishmania através de estudos por simulação computacional.

Nesse sentido, a química computacional vem se mostrando eficiente em analises de afinidade molecular entre compostos orgânicos ou sintéticos e alvos estratégicos de patógenos através do docking molecular9. Uma ferramenta promissora em predizer a melhor orientação de ajuste de encaixe de um ligante em uma proteína alvo, permitindo observar o comportamento dos compostos no sítio ativo de ligação dessas proteínas, assim como, elucidar as interações moleculares geradas pelo acoplamento. Este processo envolve predição da conformação, posição e orientação do ligante nos sítios de ligação e analise da afinidade molecular ${ }^{10}$.

Estes testes auxiliam na descoberta de novos inibidores de proteínas estratégicas de patógenos, pois avalia a afinidade molecular de compostos ligados a proteínas alvo, e identifica os tipos de interações geradas no local de ligação. Desta forma, auxilia na escolha de candidatos promissores para testes em laboratórios economizando tempo e reagentes que seriam desperdiçados ${ }^{11}$. Assim, este estudo teve como objetivo realizar uma predição computacional de alvos moleculares de Leishmania para um complexo metálico de rutênio com epiisopiloturina e óxido nítrico $\left(\right.$ Epiruno $\left._{2}\right)$.

\section{Materiais e Métodos}

\section{Obtenção da geometria molecular tridimensional do Epiruno ${ }_{2}$ empregando-se de cálculos quânticos computacionais}

A estrutura química do Epiruno ${ }_{2}$ foi desenhada utilizando-se o software Gauss View 5.012, gerando as matrizes de coordenadas cartesianas correspondentes, para que seja gerado o arquivo de entrada e ser realizado os cálculos quânticos computacionais ${ }^{13}$. Uma vez gerado o arquivo de entrada, os cálculos foram submetidos no software Gaussian $09 W^{14}$, onde os cálculos de otimização da geometria molecular e de frequências vibrações da estrutura foram realizados empregandose o método funcional DFT B3LYP, do conjunto de funções de base $6-311 \mathrm{G}^{++}(\mathrm{d}, \mathrm{p})$, com o intuito de obter a geometria molecular tridimensional que seja mais próximo possível da realidade.

\section{Interações moleculares entre os alvos de L. major com o Epiruno, por Docking molecular}

As estruturas em 3D dos alvos de L. major foram obtidas no banco de dados de proteínas $P D B$ - Protein Data Bank $^{15}$, onde foram selecionadas as proteínas mais atrativas em estudos com complexos metálicos. Em seguida, as proteínas alvos selecionadas foram preparadas com a remoção de todas as moléculas de água e outros grupos, como íons, empregando-se o software CHIMERA v.13.1 ${ }^{16}$.

$\mathrm{O}$ processo de docking molecular foi realizado empregando-se o software Autodock Tools (ADT) versão 1.5. $6^{17}$. O protocolo de Rocha et al. ${ }^{18}$ Para docking molecular com estruturas metálicas foi referência para este estudo. As proteínas alvos foram consideradas rígidas, enquanto que o Epiruno, foi considerado flexível. As cargas parciais Gasteiger ${ }^{19}$ foram calculadas após a adição de todos os hidrogênios. Os átomos de hidrogênio não polares de proteínas e ligantes foram subsequentemente mesclados. Foi gerada uma caixa cúbica de $60 \times 60 \times 60$ pontos com resolução de 0,375 $\AA$ entre os pontos da grade para todo o alvo da proteína. Os centros das grades de afinidade molecular foram definidos a partir das coordenadas do sítio ativo de cada proteína alvo selecionada.

O algoritmo genético Lamarckiano (LGA) com busca global e pseudo-Solis e Wets com busca local foram os métodos utilizados no processo de docking 
molecular ${ }^{20}$. Cada simulação consistiu de 100 corridas independentes. O restante dos parâmetros de docking foi definido com os valores padrões ${ }^{21,22}$.

\section{Resultados e Discussão}

A glicoproteína GP63 ou leishmanolisina (11ml) representa mais de $1 \%$ da proteína total do parasito de Leishmania, tendo em vista que a $1 \mathrm{lml}$ é uma metaloprotease que predomina grupos funcionais em seu sítio ativo, torna-se um alvo atrativo em estudos de atividade antileishmania ${ }^{23}$. O docking molecular entre o Epiruno ${ }_{2}$ e a proteína alvo $1 \mathrm{lml}$, resultou em uma energia de $\mathrm{G}_{\text {bind }}$ a de $-8,05 \mathrm{Kcal}^{\mathrm{mol}}{ }^{-1}$ e uma constante de inibição de 1,26 $\mu \mathrm{M}$ (Tabela 1). O complexo gerado formou quatro pontes de hidrogênio nos resíduos de aminoácidos Glu265, His264, His268 e His334, onde destacamos que além de apresentar afinidade molecular, as interações por ligações de hidrogênio são os locais em que agem as maiores forças intermoleculares deste complexo (Figura 1).

A proteína Dihidroorotate dehydrogenase ou DHODH (4ef8) possui poucos análogos de pirimidina como inibidores baseado em substrato que ligam no sítio ativo de diidroorotato ${ }^{24}$. Os inibidores conhecidos são ligados a DHODH de classe II, que são ligadas em um cofator de ubiquinina ${ }^{25}$. A maioria dos inibidores de DHODH de classe II, não é eficaz contra a DHODH de classe IA, já que seu sítio de ligação é a quinona. Esses inibidores específicos apenas agem especificamente o DHODH de classe IA Lactococcus lactis e T. brucei e análogos de orotato que apresenta inibição contra $T$. cruzi e L. major $26,27,28$.

$\mathrm{O}$ Epiruno $_{2}$ apresentou bons resultados com esta proteína, obtendo atividade inibitória frente à DHODH de $L$. major de classe IA com uma constante de inibição de 2,17 $\mu \mathrm{M}$, e uma energia de $\mathrm{G}_{\text {bind }}$ a de $-7,73 \mathrm{Kcal}$. $\mathrm{mol}^{-1}$ (Tabela 01). As interações mais eletronegativas acontecem nos aminoácidos Arg105 e Asn133, onde são

Tabela 1. Parâmetros de afinidade molecular do Epiruno $_{2}$ com as proteínas alvos de L. major.

\begin{tabular}{|c|c|c|c|c|c|}
\hline $\begin{array}{c}\text { Complexo } \\
\text { (proteína- } \\
\text { ligante) }\end{array}$ & $\begin{array}{c}\Delta \mathbf{G}_{\mathrm{bind}}{ }^{\mathrm{a}} \\
(\mathrm{kcal} . \mathrm{m} \\
\left.\mathrm{ol}^{-1}\right)\end{array}$ & $\mathbf{K i}^{\mathbf{b}}(\mu \mathbf{M})$ & $\begin{array}{c}\text { Número de } \\
\text { Corridas de } \\
\text { Encaixe } \\
\text { Independentes }\end{array}$ & $\begin{array}{c}\text { Número de } \\
\text { Conformações } \\
\text { no Primeiro } \\
\text { Cluster }\end{array}$ & $\begin{array}{c}\text { Aminoácidos } \\
\text { que } \\
\text { Interagem } \\
\text { Através de } \\
\text { Ligações de } \\
\text { Hidrogênio }\end{array}$ \\
\hline Epiruno $_{2} / 1 \mathrm{lml}$ & $-8,05$ & $1,26 \mu \mathrm{M}$ & 100 & 8 & $\begin{array}{l}\text { Glu265, } \\
\text { His264, } \\
\text { His268, } \\
\text { His } 334\end{array}$ \\
\hline Epiruno $_{2} / 4 \mathrm{ef} 8$ & $-7,73$ & $2,17 \mu \mathrm{M}$ & 100 & 7 & $\begin{array}{l}\text { Arg105, } \\
\text { Asn133 }\end{array}$ \\
\hline Epiruno $_{2} / 5142$ & $-7,62$ & $2,6 \mu \mathrm{M}$ & 100 & 7 & Lys 244 \\
\hline Epiruno $_{2} / 3$ ogz & $-6,71$ & $12,16 \mu \mathrm{M}$ & 100 & 5 & Lys 134 \\
\hline Epiruno $_{2} / 3$ tue & $-6,58$ & $14,91 \mu \mathrm{M}$ & 100 & 27 & $\begin{array}{c}\text { Asp47, Phe50, } \\
\text { Val51 }\end{array}$ \\
\hline Epiruno $_{2} / 2 \mathrm{xe} 4$ & $-6,12$ & $32,8 \mu \mathrm{M}$ & 100 & 1 & $\begin{array}{c}\text { Ala80, Asp78, } \\
\text { Leu82 }\end{array}$ \\
\hline
\end{tabular}

Nota: Epiruno 2 - Complexo de rutênio com epiisopiloturina e óxido nítrico; $11 \mathrm{lml}$ glicoproteina GP63 ou leishmanolisina; 4ef8 - Dihidroorotate dehydrogenase; 5142 Pteridine reductase 1; 3ogz - UDP-sugar pyrophosphorylase; 3tue - tryparedoxin peroxidase; 2 xe4 - oligopeptidase $B$. 


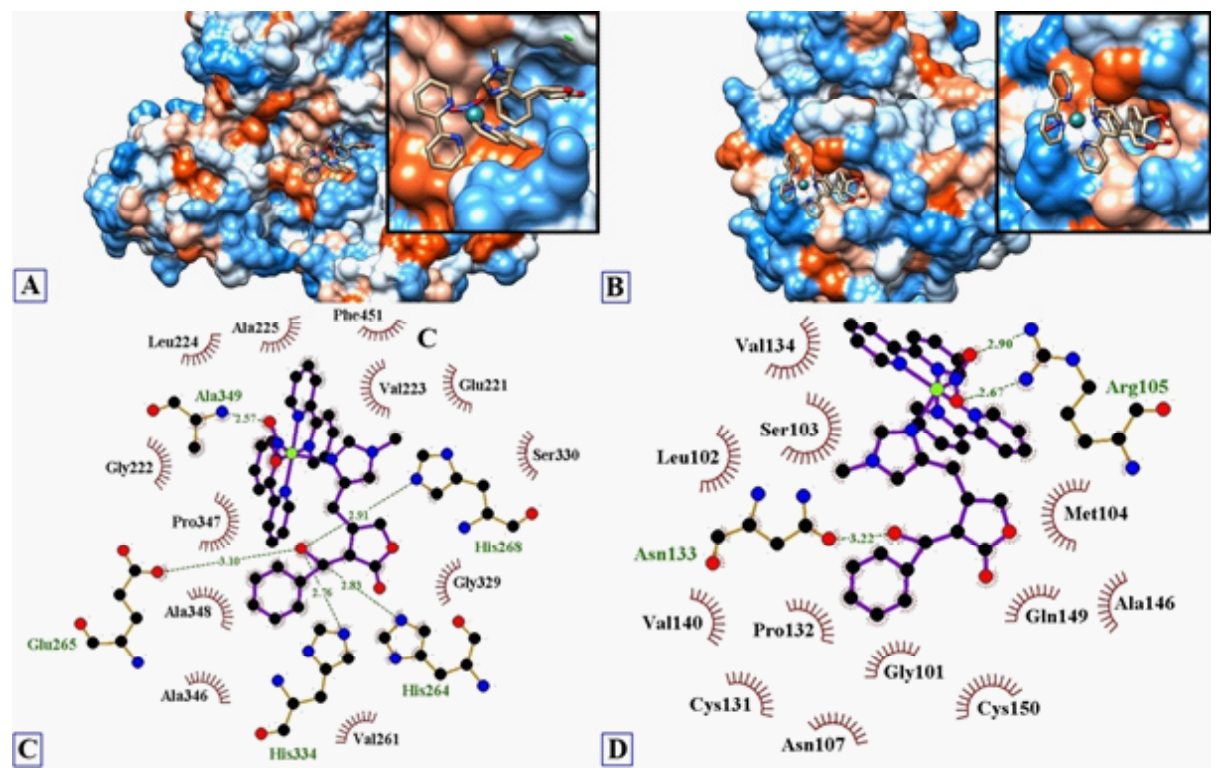

Figura 1. Docking molecular entre o Epiruno ${ }_{2}$ e as proteínas alvo $1 \mathrm{lml}$ e 4 ef8.

Nota: A) ancoragem no sítio ativo da proteína alvo $1 \mathrm{lml}$; B) ancoragem no sítio ativo da proteína alvo 4ef8; C) interação molecular entre o $11 \mathrm{ml} \mathrm{e} \mathrm{o} \mathrm{Epiruno}{ }_{2}$; D) interação molecular entre o 4ef8 e o Epiruno ${ }_{2}$.

localizadas as pontes de hidrogênio, locais de maiores forças intermoleculares entre o DHODH de L. major de classe IA e o Epiruno ${ }_{2}$ (Figura 01).

$\mathrm{O}$ docking molecular do Epiruno ${ }_{2}$ e a proteína Pteridine reductase 1 de L. major ou LmPTR1 (5142), obteve uma energia de $\mathrm{G}_{\text {bind }}{ }^{\text {a }}$ de $-7,62 \mathrm{Kcal} \mathrm{mol}^{-1}$ e uma constante de inibição de $2,6 \mu \mathrm{M}$. O complexo gerado formou uma ligação de hidrogênio no aminoácido Lys244 (Tabela 1), local onde a força intermolecular do complexo é mais intensa. A LmPTR1 é um alvo atrativo para descobertas de drogas antitrypanosomal e antileishmanial ${ }^{29}$. A literatura mostra diferentes arcabouços, como pteridina, pirrolopirimidina e benzimidazol, onde relata que a ligação inibitória da atividade da PTR1 é no biopterina ${ }^{30,31,32,33}$.

A proteína USP é o homólogo de artrópodes do receptor $\mathrm{X}$ retinóide de vertebrados $(\mathrm{RXR} \alpha, \beta$, y; NR2B1, 2, 3), moluscos e cnidários ${ }^{34}$. A USP também é uma proteína especialista em diferentes tipos de ligações ao DNA classificada como heterodimerização, que é uni presente para NRs classe $\mathrm{II}^{35}$. Em L. major, a UGP leva a um fenótipo moderado associado à atividade residual da UGP, que ocorre devido a USP ${ }^{36}$. A estrutura das UGPs possui um arranjo estrutural comum que adotam uma estrutura tripartida com um pequeno domínio catalítico central, onde possui uma dobra em sanduíche $\alpha / \beta / \alpha$ semelhante à Rossmann e um domínio C-terminal em hélice $\beta^{37,38}$.

As UGPs de L. major e de plantas são semelhantes em sua ativação, ambas são ativas como monômeros ${ }^{39}$, que após a ligação do Epiruno $_{2}$ a proteína UGP de L. major mostra alterações em sua conformidade envolvendo o domínio $\mathrm{C}$-terminal em direção ao sítio ativo e rearranjos em alças funcionais do domínio catalítico $^{40}$ como observados na Figura 2. Os resultados residuais do docking molecular da proteína UDPsugar pyrophosphorylase de L. major (3ogz) com o Epiruno $_{2}$ obteve uma energia de $\mathrm{G}_{\text {bind }}$ a de $-6,71 \mathrm{Kcal}$. mol $^{-1}$ e uma constante de inibição de $12,16 \mu \mathrm{M}$ (Tabela 1). O complexo formado resultou em uma ligação de hidrogênio no resíduo de aminoácido Lys 134, local em que acontece a ligação mais intensa deste complexo. Os aminoácidos Arg127, Asp271, Gln270, Glu126, Gly122, Gly123, His224, Leu124, Leu128 e Val120 realizam as interações hidrofóbicas (Figura 2 (D)).

$\mathrm{O}$ docking molecular do tryparedoxin peroxidase de L. major (3tue) com o Epiruno ${ }_{2}$ apresentou uma energia de $\mathrm{G}_{\text {bind }}{ }^{\mathrm{a}}$ de $-6.58 \mathrm{Kcal}^{\mathrm{mol}} \mathrm{m}^{-1} \mathrm{e}$ uma constante de inibição de $14.91 \mu \mathrm{M}$ (Tabela 01), mostrando possuir atividade anti-tryparedoxin peroxidase de L. major. O 3tue tem sido um alvo atrativo para pesquisas antileishmania por conter um metabolismo hidroperóxido dependente de tripanotiona, pois além desse parasita não conter catalase, também possui peroxidases dependentes de selênio, redutase da glutationa e da tioredoxina redutase e por possuir uma defesa antioxidante baseada em um sistema de enzimas dependente da tripanotiona ditiol único (N1, N8-bis (glutationilo) espermidina, $\mathrm{T}$ (SH)2 $)^{41}$. Desta forma, tem levado cientistas a estudarem seu metabolismo e desenvolver novas alternativas de tratamento necessárias devido à baixa eficácia e segurança dos atuais medicamentos ${ }^{42}$. A ilustração do docking molecular do complexo Epiruno/3tue está disponível na Figura 2 (B, E).

A oligopeptidase $\mathrm{B}(\mathrm{OPB})$ é uma sirina peptidase com dibásico especificidade do substrato. Podendo ser encontrada restritamente em plantas, bactérias e patógenos tripanossomatídeos, tornando-se um fator de 

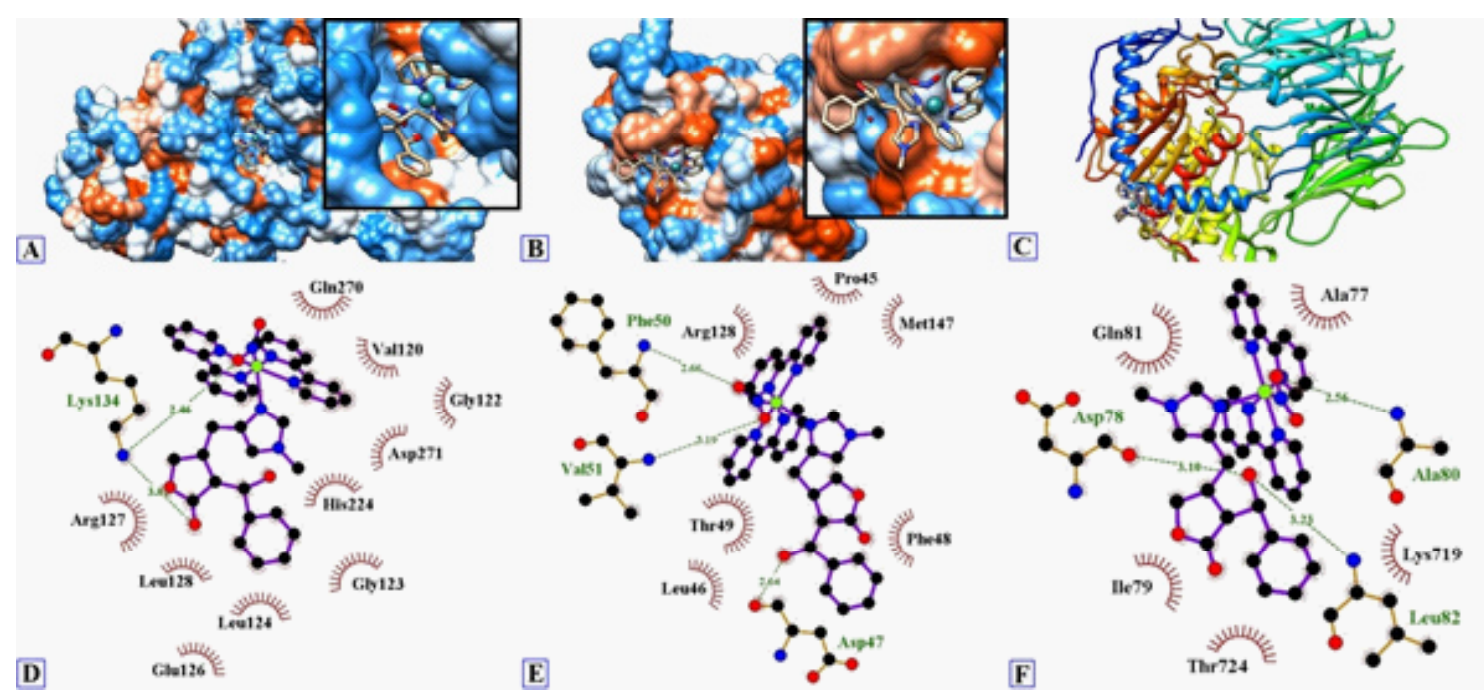

Figura 2. Docking molecular entre o Epiruno, e as proteínas alvo 3ogz, 3tue e 2xe4.

\begin{abstract}
Nota: A) ancoragem no sítio ativo da proteína alvo 3ogz; B) ancoragem no sítio ativo da proteína alvo 3tue; C) ancoragem no sítio ativo da proteína alvo 2xe4; D) interação molecular entre o $30 g z$ e o Epiruno 2 ; E) interação molecular entre o 3tue e o Epiruno ${ }_{2}$ F) interação molecular entre o 2xe4 e o Epiruno .
\end{abstract}

virulência atrativo em estudos farmacológicos ${ }^{43}$. A OPB foi identificada em diversas espécies de Leishmania incluindo as espécies de L. major e L. mexicana ${ }^{44}$. Atualmente possuem apenas três fármacos que inibem a OPB de L. major, que são: pentamidina, diminazeno e suramina $^{45,46}$. $\mathrm{O}$ Epiruno $_{2}$ se apresenta como um agente inibidor alternativo que apresentou uma interação molecular interessante clinicamente ${ }^{47}$, possuindo uma energia de $\mathrm{G}_{\text {bind }}{ }^{a}$ de $-6.12 \mathrm{Kcal}^{\mathrm{mol}}{ }^{-1}$ e uma constante de inibição de $32.8 \mu \mathrm{M}$. O complexo gerado formou três pontes de hidrogênio ligadas nos resíduos de aminoácidos Ala80, Asp78 e Leu82. Estas ligações são responsáveis pelas interações de maiores forças intermoleculares do complexo Epiruno 2 /2xe4 (Tabela 1). Os aminoácidos Ala77, Gln81, Ile79, Lys 719, Thr724 realizam as interações hidrofóbicas (Figura 2 (F)).

\section{Conclusão}

$\mathrm{O}$ composto Epiruno ${ }_{2}$ se apresentou atrativo para estudos experimentais futuros in vitro, ex vivo e in vivo, pois seus resultados in sílico apresentaram boas interações moleculares para todas as proteínas tridimensionais de L. major analisadas neste estudo. Destacando-se o complexo Epiruno $/ 11 \mathrm{lml}$ gerado, que resultou em uma excelente afinidade molecular com

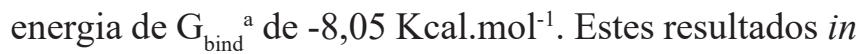
sílico apresentam o Epiruno ${ }_{2}$ como possível candidato a fármaco contra a leishmaniose, porém este composto deve ser submetido a testes experimentais para viabilizar e comprovar sua atividade leishmanicida apresentada neste estudo de predição computacional.

\section{Referências}

1. WHO. Control of Leishmaniasis Report of a meeting of the WHO Committee of Experts on the Control of Leishmaniasis. 2019. Available from: http://www.who.int/mediacentre/factsheets/fs375/en/

2. Theuretzbacher U. Future antibiotics scenarios: is the tide starting to turn? International Journal of Antimicrobial Agents [Internet]. Elsevier BV; 2009;34(1):15-20. Available from: http://dx.doi.org/10.1016/j. ijantimicag.2009.02.005

3. Araújo JL, Santos GT, Sousa LA de, Santos GT, Silva WDF, Sousa ADO, et al. Molecular docking of rutenum complex with epiisopyloturin and nitric oxide against nucleoside diphosphate kinase protein Leishmania. Research, Society and Development [Internet]. 2020;9(2):59922121. Available from: http://dx.doi.org/10.33448/rsd-v9i2.2121

4. Khamesipour A. Therapeutic vaccines for leishmaniasis. Expert Opinion on Biological Therapy [Internet]. Informa Healthcare; 2014;14(11):1641-9. Available from: http://dx.doi.org/10.1517/14712598.2014.945415

5. Colotti G, Ilari A, Fiorillo A, Baiocco P, Cinellu MA, Maiore L, et al. Metal-Based Compounds as Prospective Antileishmanial Agents: Inhibition of Trypanothione Reductase by Selected Gold Complexes. ChemMedChem [Internet]. 2013;n/a-n/a. Available from: http://dx.doi.org/10.1002/ cmdc. 201300276

6. Baiocco P, Ilari A, Ceci P, Orsini S, Gramiccia M, Di Muccio T, et al. Inhibitory Effect of Silver Nanoparticles on Trypanothione Reductase Activity and Leishmania infantum Proliferation. ACS Medicinal Chemistry Letters [Internet]. 2010;2(3):230-3. Available from: http://dx.doi.org/10.1021/ ml1002629

7. Otero L, Vieites M, Boiani L, Denicola A, Rigol C, Opazo L, et al. Novel Antitrypanosomal Agents Based on Palladium Nitrofurylthiosemicarbazone Complexes: DNA and Redox Metabolism as Potential Therapeutic Targets $\dagger$. Journal of Medicinal Chemistry [Internet]. 2006;49(11):3322-31. Available from: http://dx.doi.org/10.1021/jm0512241

8. Bonse S, Richards JM, Ross SA, Lowe G, Krauth-Siegel RL. (2,2`:6‘ $2^{`}$ ‘ '-Terpyridine)platinum(II) Complexes Are Irreversible Inhibitors ofTrypanosomacruziTrypanothione Reductase But Not of Human Glutathione Reductase. Journal of Medicinal Chemistry [Internet]. 2000;43(25):4812-21. Available from: http://dx.doi.org/10.1021/jm000219o

9. Meng X-Y, Zhang H-X, Mezei M, Cui M. Molecular Docking: A Powerful Approach for Structure-Based Drug Discovery. Current Computer AidedDrug Design [Internet]. 2011;7(2):146-57. Available from: http://dx.doi. 


\section{org/10.2174/157340911795677602}

10. Morris GM, Lim-Wilby M. Molecular Docking. Molecular Modeling of Proteins [Internet]. 2008;365-82. Available from: http://dx.doi. org/10.1007/978-1-59745-177-2_19

11. Figueiredo KA, Figueiredo JFS, Costa RKM, Alves MMM, Magalhães JL, Carvalho ALM, et al. Prospecting Biochemical Targets for in silico Study for Antileishmania Chemotherapy. Revista Virtual de Química [Internet]. 2018;10(5):1485-501. Available from: http://dx.doi.org/10.21577/19846835.20180101

12. Dennington RD, Keith TA, Millan JM. GaussView, v. 5.0.8, Semicchem, Inc.: Shawnee KS, 2008.

13. Araújo JL, Sousa AO, Bastos RS, Santos GT, Araújo JL, Rocha JA. Estudo in sílico da atividade biológica por docagem molecular da desloratadina contra esquistossomose. Revista Eletrônica Acervo Saúde [Internet]. 2019;28(sup):e993. Available from: https://doi.org/10.25248/reas. e993.2019

14. Frisch MJ, Trucks GW, Schlegel HB, Scuseria GE, Robb MA, Cheeseman JR, et al. Gaussian09, Revision C.01; Gaussian, Inc.: Wallingford, CT, 2010 .

15. Berman HM. The Protein Data Bank. Nucleic Acids Research [Internet]. 2000;28(1):235-42. Available from: http://dx.doi.org/10.1093/nar/28.1.235

16. Pettersen EF, Goddard TD, Huang CC, Couch GS, Greenblatt DM, Meng EC, et al. UCSF Chimera-A visualization system for exploratory research and analysis. Journal of Computational Chemistry [Internet]. 2004;25(13):160512. Available from: http://dx.doi.org/10.1002/jcc.20084

17. Morris GM, Huey R, Olson AJ. Using AutoDock for Ligand-Receptor Docking. Current Protocols in Bioinformatics [Internet]. 2008;24(1). Available from: http://dx.doi.org/10.1002/0471250953.bi0814s24

18. Rocha JA, Rego NCS, Carvalho BTS, Silva FI, Sousa JA, Ramos $\mathrm{RM}$, et al. Computational quantum chemistry, molecular docking, and ADMET predictions of imidazole alkaloids of Pilocarpus microphyllus with schistosomicidal properties. Chen M, editor. PLOS ONE [Internet]. 2018;13(6):e0198476. Available from: http://dx.doi.org/10.1371/journal. pone. 0198476

19. Gasteiger J, Marsili M. Iterative partial equalization of orbital electronegativity-a rapid access to atomic charges. Tetrahedron [Internet]. 1980;36(22):3219-28. Available from: http://dx.doi.org/10.1016/00404020(80)80168-2

20. Solis FJ, Wets RJ-B. Minimization by Random Search Techniques. Mathematics of Operations Research [Internet]. 1981;6(1):19-30. Available from: http://dx.doi.org/10.1287/moor.6.1.19

21. Morris GM, Goodsell DS, Halliday RS, Huey R, Hart WE, Belew RK, et al. Automated docking using a Lamarckian genetic algorithm and an empirical binding free energy function. Journal of computational chemistry [Internet]. 1998;19(14):1639-1662. Available from: https://doi.org/10.1002/ (SICI)1096-987X(19981115)19:14\%3C1639::AID-JCC10\%3E3.0.CO;2-B

22. Ramos RM, Perez JM, Baptista LA, de Amorim HLN. Interaction of wild type, G68R and L125M isoforms of the arylamine-N-acetyltransferase from Mycobacterium tuberculosis with isoniazid: a computational study on a new possible mechanism of resistance. Journal of Molecular Modeling [Internet]. 2012;18(9):4013-24. Available from: http://dx.doi.org/10.1007/s00894-0121383-6

23. Jaffe CL, Dwyer DM. Extracellular release of the surface metalloprotease, gp63, from Leishmania and insect trypanosomatids. Parasitology Research [Internet]. 2003;91(3):229-37. Available from: http://dx.doi.org/10.1007/ s00436-003-0960-0

24. DeFrees SA, Sawick DP, Cunningham B, Heinstein PF, James Morré D, Cassady JM. Structure-activity relationships of pyrimidines as dihydroorotate dehydrogenase inhibitors. Biochemical Pharmacology [Internet]. 1988;37(20):3807-3816. Available from: http://dx.doi.org/10.1016/00062952(88)90060-3

25. K. Vyas V, Ghate M. Recent Developments in the Medicinal Chemistry and Therapeutic Potential of Dihydroorotate Dehydrogenase (DHODH) Inhibitors. Mini-Reviews in Medicinal Chemistry [Internet]. 2011;11(12):1039-55. Available from: http://dx.doi.org/10.2174/138955711797247707
26. Palfey BA, Björnberg O, Jensen KF. Specific Inhibition of a Family 1A Dihydroorotate Dehydrogenase by Benzoate Pyrimidine Analogues. Journal of Medicinal Chemistry [Internet]. 2001;44(18):2861-4. Available from: http://dx.doi.org/10.1021/jm015535x

27. Arakaki TL, Buckner FS, Gillespie JR, Malmquist NA, Phillips MA, Kalyuzhniy O, et al. Characterization of Trypanosoma brucei dihydroorotate dehydrogenase as a possible drug target; structural, kinetic and RNAi studies. Molecular Microbiology [Internet]. 2008;68(1):37-50. Available from: http:// dx.doi.org/10.1111/j.1365-2958.2008.06131.x

28. Cheleski J, Rocha JR, Pinheiro MP, Wiggers HJ, da Silva ABF, Nonato $\mathrm{MC}$, et al. Novel insights for dihydroorotate dehydrogenase class $1 \mathrm{~A}$ inhibitors discovery. European Journal of Medicinal Chemistry [Internet]. 2010;45(12):5899-909. Available from: http://dx.doi.org/10.1016/j. ejmech.2010.09.055

29. Araújo JL, Bastos RS, Santos GT, Araújo JL, Rocha JA. Evaluation by molecular docking of inhibitors of the enzyme pteridine reductase 1 from leishmania. Revista Prevenção de Infecção e Saúde [Internet]. 2019;5. Available from: http://dx.doi.org/10.26694/repis.v5i0.9056

30. Cavazzuti A, Paglietti G, Hunter WN, Gamarro F, Piras S, Loriga M, et al. Discovery of potent pteridine reductase inhibitors to guide antiparasite drug development. Proceedings of the National Academy of Sciences [Internet]. 2008;105(5):1448-1453. Available from: http://dx.doi.org/10.1073/ pnas. 0704384105

31. Tulloch LB, Martini VP, Iulek J, Huggan JK, Lee JH, Gibson CL, et al. Structure-Based Design of Pteridine Reductase Inhibitors Targeting African Sleeping Sickness and the Leishmaniases. Journal of Medicinal Chemistry [Internet]. 2010;53(1):221-229. Available from: http://dx.doi.org/10.1021/ jm901059x

32. Mpamhanga CP, Spinks D, Tulloch LB, Shanks EJ, Robinson DA, Collie IT, et al. One Scaffold, Three Binding Modes: Novel and Selective Pteridine Reductase 1 Inhibitors Derived from Fragment Hits Discovered by Virtual Screening. Journal of Medicinal Chemistry [Internet]. 2009;52(14):44544465. Available from: http://dx.doi.org/10.1021/jm900414x

33. Khalaf AI, Huggan JK, Suckling CJ, Gibson CL, Stewart K, Giordani F, et al. Structure-Based Design and Synthesis of Antiparasitic Pyrrolopyrimidines Targeting Pteridine Reductase 1. Journal of Medicinal Chemistry [Internet]. 2014;57(15):6479-6494. Available from: http://dx.doi. org/10.1021/jm500483b

34. Laudet V, Gronemeyer H. PXR. The Nuclear Receptor FactsBook [Internet]. 2002;220-226. Available from: http://dx.doi.org/10.1016/b978$012437735-6 / 50022-9$

35. Brelivet Y, Kammerer S, Rochel N, Poch O, Moras D. Signature of the oligomeric behaviour of nuclear receptors at the sequence and structural level. EMBO reports [Internet]. 2004;5(4):423-429. Available from: http:// dx.doi.org/10.1038/sj.embor.7400119

36. Lamerz A-C, Damerow S, Kleczka B, Wiese M, van Zandbergen G, Lamerz J, et al. Deletion of UDP-glucose pyrophosphorylase reveals a UDPglucose independent UDP-galactose salvage pathway in Leishmania major. Glycobiology [Internet]. 2010;20(7):872-882. Available from: http://dx.doi. org/10.1093/glycob/cwq045

37. Aragao D, Fialho AM, Marques AR, Mitchell EP, Sa-Correia I, Frazao C. The Complex of Sphingomonas elodea ATCC 31461 Glucose-1-Phosphate Uridylyltransferase with Glucose-1-Phosphate Reveals a Novel Quaternary Structure, Unique among Nucleoside Diphosphate-Sugar Pyrophosphorylase Members. Journal of Bacteriology [Internet]. 2007;189(12):4520-4528. Available from: http://dx.doi.org/10.1128/jb.00277-07

38. Roeben A, Plitzko JM, Körner R, Böttcher UMK, Siegers K, HayerHartl M, et al. Structural Basis for Subunit Assembly in UDP-glucose Pyrophosphorylase from Saccharomyces cerevisiae. Journal of Molecular Biology [Internet]. 2006;364(4):551-560. Available from: http://dx.doi. org/10.1016/j.jmb.2006.08.079

39. Lamerz A-C, Haselhorst T, Bergfeld AK, von Itzstein M, GerardySchahn R. Molecular Cloning of theLeishmania majorUDP-glucose Pyrophosphorylase, Functional Characterization, and Ligand Binding Analyses Using NMR Spectroscopy. Journal of Biological Chemistry [Internet]. 2006;281(24):16314-16322. Available from: http://dx.doi. org/10.1074/jbc.m600076200

40. Steiner T. Open and Closed Structures of the UDP-Glucose 
Pyrophosphorylase from Leishmania major. Worldwide Protein Data Bank. 2007. Available from: http://dx.doi.org/10.2210/pdb2oef/pdb

41. Flohé L. The trypanothione system and the opportunities it offers to create drugs for the neglected kinetoplast diseases. Biotechnology Advances [Internet]. 2012;30(1):294-301. Available from: http://dx.doi.org/10.1016/j. biotechadv.2011.05.012

42. Maltezou HC. Drug Resistance in Visceral Leishmaniasis. Journal of Biomedicine and Biotechnology [Internet]. 2009;2010:1-8. Available from: http://dx.doi.org/10.1155/2010/617521

43. Burleigh BA, Woolsey AM. Cell signalling andTrypanosoma cruziinvasion. Cellular Microbiology [Internet]. 2002;4(11):701-711. Available from: http://dx.doi.org/10.1046/j.1462-5822.2002.00226.x

44. Ouakad M, Chenik M, Ben Achour-Chenik Y, Louzir H, Dellagi K. Gene expression analysis of wild Leishmania major isolates: identification of genes preferentially expressed in amastigotes. Parasitology Research [Internet]. 2006;100(2):255-264. Available from: http://dx.doi.org/10.1007/ s00436-006-0277-x

45. Morty RE, Troeberg L, Pike RN, Jones R, Nickel P, Lonsdale-Eccles $\mathrm{JD}$, et al. A trypanosome oligopeptidase as a target for the trypanocidal agents pentamidine, diminazene and suramin. FEBS Letters [Internet]. 1998;433(3):251-256. Available from: http://dx.doi.org/10.1016/s00145793(98)00914-4

46. McLuskey K, Paterson NG, Bland ND, Isaacs NW, Mottram JC. Crystal Structure ofLeishmania majorOligopeptidase B Gives Insight into the Enzymatic Properties of a Trypanosomatid Virulence Factor. Journal of Biological Chemistry [Internet]. 2010;285(50):39249-39259. Available from: http://dx.doi.org/10.1074/jbc.m110.156679

47. Araújo JL, Bastos RS, Santos GT, Alves MMM, Figueiredo KA, Sousa LA, et al. Molecular docking and evaluation of antileishmania activity of a ruthenium complex with epiisopiloturine and nitric oxide. Journal of Biosciences and Medicines [Internet]. 2020; 8(5):42-53. Available from: https://doi.org/10.4236/jbm.2020.85005. 